\title{
CORRESPONDENCE
}

\section{Simvastatin-induced effects on bronchial epithelial cells from COPD donors}

\section{To the Editor:}

I read with interest the report by LeE et al. [1] on inhibition by simvastatin of double-stranded (ds)RNA-induced bronchial epithelial cell production of RANTES, with supportive in vivo data in mice. dsRNA is an interesting challenge agent. It not only mimics viral infection, as noted by LEE et al. [1], but may also reflect effects of released mRNA or secondary structure from necrotic cells that commonly occur in severe bronchial diseases (for references, see [2]). RANTES, similar to interferon (IFN)- $\beta$, is induced by activation of the transcription factor IRF3 (IFN regulatory factor 3) [3]. Hence, the anti-RANTES effect demonstrated by LEE et al. [1] may agree with our current demonstration of simvastatin-induced inhibition of IRF3 phosphorylation in dsRNA-challenged bronchial epithelial cells; we used primary cells obtained by bronchial brushings in chronic obstructive pulmonary disease (COPD) patients [4]. We further showed that the inhibition of IRF3 activation occurred without effects on nuclear factor (NF)-кB. As expected then, simvastatin inhibited IFN- $\beta$ gene expression and protein production without reducing NF-kB-dependent cytokines, such as tumour necrosis factor- $\alpha$ and CXCL8 [4]. Inhibition of IFN- $\beta$, a major antiviral IFN, needs consideration by investigators who may wish to explore anti-RANTES effects of simvastatin in patients with respiratory diseases.

I agree on the importance of finding pharmacological explanations for the intriguing effects on inflammatory disease development that serendipitously have been associated with statin treatment. However, in general, the concentrations of simvastatin which are anti-inflammatory in vitro $([1,4]$ and references cited therein) are approximately 20-times larger than the therapeutic blood levels [4]. This fact casts doubt on the direct clinical relevance of the in vitro cell data, except for the tentative use of very large statin doses in terminally ill individuals. LEE et al. [1] successfully used the purely experimental, topical airway route for simvastatin administration to mice in vivo. However, they did not discuss the concentration issue; indeed, it is rarely discussed in current literature [4]. On this note, the relatively high challenge concentration of dsRNA (polyinosinic-polycytidylic acid, $25 \mu \mathrm{g} \cdot \mathrm{mL}^{-1}$ ) used by LEE et al. [1] also needs validation to exclude toxic cell effects.

We became interested in simvastatin's epithelial actions mainly because it effectively inhibited dsRNA-induced production of thymic stromal lymphopoietin (TSLP) [4]. TSLP is an upstream cytokine that is overexpressed in severe asthma and COPD [5]. TSLP is also overexpressed and overproduced in response to dsRNA and rhinoviral infection in bronchial epithelial cells obtained from donors with asthma and COPD, respectively ([2] and references cited therein). Based on these observations and on the rapidly evolving roles of TSLP in immunopathogenesis, ranging from switching on T-helper cell-2 type inflammation to being causally involved in autoimmunity-driven inflammation (references cited in [4]), we have suggested that TSLP may be important in exacerbations and development of severe stages of COPD and asthma $[2,4]$. We further discovered that IRF3, rather than the previously acknowledged role of NF- $\mathrm{KB}$, was involved in the generation and inhibition of TSLP in diseased bronchial epithelial cells [4].

I would challenge the view, inferred by LEE et al. [1], that simvastatin-induced inhibition of RANTES is a class effect of statins. Statin drugs have been developed based on their capacity for inhibiting HMG-CoA (3-hydroxy-3-methylglutarylcoenzyme A) reductase that produces mevalonic acid. Mevalonic acid is involved both in cholesterol synthesis and in the synthesis of the isoprenoid intermediates, farnesyl and geranyl pyrophosphates, which are linked to inflammation through Ras and Rho small G-protein activation: Rho-GTPases activate NF- $\mathrm{KB}$ which is involved in the generation of cytokines. Indeed, most pleiotropic anti-inflammatory effects of statins have been mevalonate-dependent and reported to reflect inhibition of NF-kB (for references, see [4]). In contrast, we demonstrated in bronchial epithelial cells from COPD donors that simvastatin's "anti-inflammatory" effects involved inhibition of IRF3 activation, and were independent of both mevalonate and NF- $\mathrm{KB}$ [4]. It would be of interest to learn whether the anti-RANTES action of simvastatin reported by LEE et al. [1] can be antagonised by exogenous mevalonate? My guess is that an appropriate concentration of mevalonate $\left(\sim 20 \mu \mathrm{g} \cdot \mathrm{mL}^{-1}\right)$ will not reduce simvastatin's effect in this case.

Interestingly, similar to simvastatin, the adapter molecule MyD88 emerges as an endogenous, selective IRF3 inhibitor, downregulating production of IFN- $\beta$ and RANTES [3]. MyD88 is essential for signalling by most Toll-like receptors (TLRs), except TLR3 for which dsRNA is the archetypical ligand. Whether MyD88 may regulate TSLP as well has not been examined. LEE et al. [1] do not report effects on IRF3, but advance the possibility that AKT and STAT3 are involved in the RANTES production that is inhibited by simvastatin [1]. It will be of interest to explore the potential interactions between IRF3 activation and STAT3 regarding bronchial epithelial actions of dsRNA and the inhibitory pharmacology of simvastatin. The promising prospect is that further revelations in this field may lead to discovery of novel drugs for treatment of severe respiratory diseases. 


\section{Lena Uller}

Unit of Respiratory Immunopharmacology, Dept Experimental Medical Science, Lund University, Lund, Sweden.

Correspondence: L. Uller, Unit of Respiratory Immunopharmacology, Dept Experimental Medical Science, BMC D12, Lund University, 22184 Lund, Sweden. E-mail: lena.uller@ med.lu.se

Statement of Interest: None declared.

\section{REFERENCES}

1 Lee CS, Yi EH, Lee JK, et al. Simvastatin suppresses RANTESmediated neutrophilia in polyinosinic-polycytidylic acid-induced pneumonia. Eur Respir J 2013; 41: 1147-1156.
2 Calvén J, Yudina Y, Hallgren $\mathrm{O}$, et al. Viral stimuli trigger exaggerated thymic stromal lymphopoietin expression by chronic obstructive pulmonary disease epithelium: role of endosomal TLR3 and cytosolic RIG-I-like helicases. J Innate Immun 2011; 4: 86-99.

3 Siednienko J, Halle A, Nagpal K, et al. TLR3-mediated IFN- $\beta$ gene induction is negatively regulated by the TLR adaptor MyD88 adaptor-like. Eur J Immunol 2010; 40: 3150-3160.

4 Brandelius A, Mahmutovic Persson I, Calvén J, et al. Selective inhibition by simvastatin of IRF3 phosphorylation and TSLP production in dsRNA-challenged bronchial epithelial cells from COPD donors. Br J Pharmacol 2013; 168: 363-374.

5 Ying S, O'Connor B, Ratoff J, et al. Expression and cellular provenance of thymic stromal lymphopoietin and chemokines in patients with severe asthma and chronic obstructive pulmonary disease. J Immunol 2008; 181: 2790-2798.

DOI: $10.1183 / 09031936.00133812$

\section{Complexities of oestradiol pharmacology in pulmonary arterial hypertension}

\section{To the Editor:}

We read with great interest the recent article by YUAN et al. [1]. The authors confirm previous observations that female sex and $\mathrm{E}_{2}$ (17 $\beta$-oestradiol) protect against a rat model of pulmonary hypertension $(\mathrm{PH})$, namely monocrotaline-induced PH (MCT$\mathrm{PH})$, and that the beneficial effects of $\mathrm{E}_{2}$ in MCT-PH are associated with increased lung nitric oxide, prostacyclin levels and reduced endothelin levels. Furthermore, based on $E_{2}$ plasma levels and altered expression of key enzymes, they suggest that $\mathrm{E}_{2}$ deficiency may play a role in MCT-PH.

We would like to draw attention to another dimension of $E_{2}$ pharmacology that adds to the complexity of $E_{2}$ in experimental $\mathrm{PH}$ and human pulmonary arterial hypertension (PAH), i.e. the metabolism of $E_{2}$ to biologically active metabolites. We also would like to discuss the concept that the overall effects of sex and $E_{2}$ in $\mathrm{PH}$ may depend on the model system.

Despite the fact that $E_{2}$ and female sex protect against MCT$\mathrm{PH}$, surprisingly $\mathrm{PAH}$ occurs more frequently in females than males. This incongruous finding is called the "PAH gender paradox" [2]. Even so, females with $\mathrm{PAH}$ have better right ventricular function and survival compared to males with PAH [3-5]. The apparent contradictions posed by the effects of female sex and oestrogens in experimental $\mathrm{PH}$ versus human $\mathrm{PAH}$ could be explained by the complexity of $\mathrm{E}_{2}$ metabolism, the opposing effects of $E_{2}$ versus its metabolites on endothelial vascular remodelling, limitations of the experimental models used, and the opposite roles that $\mathrm{E}_{2}$ may play in the pulmonary vasculature versus the right ventricle.

Several lines of evidence strongly suggest that the vascular protective effects of $\mathrm{E}_{2}$ are mediated largely by its downstream metabolites [2]. Notably, 2-methoxyestradiol (2ME, a major non-oestrogenic metabolite of $\mathrm{E}_{2}$ ) attenuates the development of and retards the progression of MCT- or hypoxia-induced $\mathrm{PH}$ in male rats and mediates the protective effects of $E_{2}$ in female rats with MCT- or bleomycin-induced PH [2]. Furthermore, $2 \mathrm{ME}$ is decisively more potent than $\mathrm{E}_{2}$ in increasing prostacyclin synthesis and release, in inhibiting endothelin synthesis in endothelial cells, in stimulating nitric oxide release from endothelium, and in inhibiting growth of systemic and pulmonary vascular smooth muscle cells [2]. Similar to the effects of $E_{2}$ in the YUAN et al. [1] study, inhibition of vascular remodelling by $2 \mathrm{ME}$ is also associated with down regulated expression of phosphorylated Akt and up regulated expression of cyclooxygenase-2 [6]. Thus, at least in part, the beneficial effects of oestradiol in MCT-PAH are mediated by $2 \mathrm{ME}$.

The hallmark vascular change in MCT-PH is medial thickening and neomuscularisation, with only mild endothelial damage that does not lead to occlusive proliferation of endothelial cells and formation of plexiform lesions. $\mathrm{E}_{2}$ and $2 \mathrm{ME}$ have opposing effects on endothelial cells: $E_{2}$ is promitogenic, proangiogenic and antiapoptotic, whereas $2 \mathrm{ME}$ is antimitogenic, antiangiogenic and proapoptotic. This may have significant ramifications for the overall effects of oestrogens in experimental $\mathrm{PH}$. We suggested that the effects of sex and oestrogens in experimental $\mathrm{PH}$ should be studied in a model of severe angio-proliferative PH (administration of vascular endothelial growth factor 2 receptor antagonist SU5416 and exposure to hypoxia) [2]. This model, despite its own limitations, more closely mimics the key vascular alterations and progressive and deadly character of disease seen in severe PAH in humans. In a recent study in this model (and contrary to the effects of sex and $E_{2}$ in the study by YUAN et al. [1]), we demonstrated that female rats develop more severe $\mathrm{PH}$ than males (numerous plexiform lesions in females compared to predominantly occlusive lesions in male rats), that in ovariectomised rats rescue treatment with $E_{2}$ exacerbates vascular lesions and 